Published in final edited form as:

J Investig Med. 2017 January ; 65(1): 43-48. doi:10.1136/jim-2016-000213.

\title{
Association between serum cotinine level and prevalence of non-alcoholic fatty liver disease: a cross-sectional study from the Third National Health and Nutrition Examination Survey
}

\author{
Huafeng Shen ${ }^{1}$, Jennifer L Peng ${ }^{2}$, Sucharat Tayarachakul ${ }^{2}$, and Suthat Liangpunsakul ${ }^{2,3,4}$ \\ ${ }^{1}$ Division of Gastroenterology and Hepatology, Department of Medicine, University of lowa, lowa \\ City, lowa, USA \\ 2Division of Gastroenterology and Hepatology, Department of Medicine, Indiana University, \\ Indianapolis, Indiana, USA \\ ${ }^{3}$ Roudebush Veterans Administration Medical Center, Indianapolis, Indiana, USA \\ ${ }^{4}$ Department of Biochemistry and Molecular Biology, Indiana University School of Medicine, \\ Indianapolis, Indiana, USA
}

\begin{abstract}
The data on the effect of smoking on non-alcoholic fatty liver disease (NAFLD) has been controversial. The aim of this study was to investigate if an association exists between serum cotinine level (a tobacco biomarker) and NAFLD prevalence in the general US population. We conducted a crosssectional analysis of data from the Third National Health and Nutrition Examination Survey (NHANES III). We included 11,003 adults aged 20-74 years who underwent ultrasonography. Of those, 4036 were identified as having NAFLD and 6967 were recognized as controls. The percentage of current smokers was significantly lower in subjects with NAFLD compared with those in controls $(21.5 \%$ vs $26.0 \%$, p $<0.01)$. After adjustment for potential confounders, there was no association between current or former smokers with NAFLD. Additionally, no associations were observed between the levels of serum cotinine and NAFLD. No association between serum cotinine levels at each quartile level and NAFLD was observed regardless of smoking status. In this large US population-based study, we did not find an association between NAFLD and self-reported smoking status or measured serum cotinine level.
\end{abstract}

\footnotetext{
Correspondence to: Dr Suthat Liangpunsakul, Associate Professor of Medicine, Biochemistry and Molecular Biology, Division of Gastroenterology and Hepatology, 550 N. University Blvd, UH 4100, Indianapolis, IN 46202, USA; sliangpu@iupui.edu.

Contributors: HS was involved in study conception, design, data gathering, and analyses. All contributed to the preparation of the manuscript. All have made a substantial contribution to the writing and intellectual content of the article and acknowledge they have exercised due care in ensuring the integrity of the work. All authors approved the final version of the manuscript.

Competing interests: None declared.

Ethics approval: NCHS Research Ethics Review Board.

Provenance and peer review: Not commissioned; externally peer reviewed.
} 


\section{INTRODUCTION}

Non-alcoholic fatty liver disease (NAFLD) is one of the most common chronic liver diseases worldwide. ${ }^{1}$ It comprises the spectrum of fatty liver diseases including simple steatosis, steatohepatitis, and cirrhosis. ${ }^{1}$ In a sense, NAFLD represents another component of metabolic syndrome (MetS) ${ }^{23}$ since not only is obesity a shared major risk factor but decreased insulin sensitivity is also commonly observed in both. Additionally, the strong association of NAFLD with features of MetS ${ }^{4}$ such as obesity, central fat distribution, diabetes, dyslipidemia, and hypertension, provide further support for this hypothesis. Several reports and a meta-analysis have demonstrated smoking contributes to MetS. ${ }^{5-7}$ Slagter et a $P^{7}$ investigated the relationship between smoking and the individual components of MetS. The most notable differences between smokers and non-smokers were observed in the levels of high-density lipoprotein (HDL) and triglycerides. This finding was seen in men and women, irrespective of their body mass index (BMI). ${ }^{7}$ Since MetS is a known risk factor for NAFLD, several studies were conducted to determine the association between smoking and NAFLD. However, the results have been conflicting. ${ }^{89}$ In a community-based study of 8580 Chinese subjects, the authors found that both passive and heavy active smoking were associated with NAFLD. ${ }^{9}$ Conversely, no association was observed in a cross-sectional study from Mexico in which the routine health examination results from 933 subjects were examined. ${ }^{8}$

A plausible explanation for these contradicting results could lie in the reliability of the questionnaire used to determine smoking status. ${ }^{1011}$ Smoke exposure can be assessed with levels of serum cotinine, which is a metabolite of nicotine often applied as a surrogate measurement of smoke exposure due to its longer half-life than nicotine. ${ }^{12}$ The half-life of cotinine is about 24 hours compared with nicotine's half-life of $30 \mathrm{~min} .{ }^{12}$ Its levels, when use with in combination with self-reported status, can confirm the history of smoke exposure.

As part of the Third National Health and Nutrition Examination Survey (NHANES III), ultrasonographic examinations of the abdomen were performed and the presence or absence of hepatic steatosis was determined through a standardized algorithm. ${ }^{13}$ Given the conflicting association between NAFLD and smoking as demonstrated by the aforementioned self-reported questionnaires, we conducted this population-based study using data from NHANES III, with a focus on levels of serum cotinine, to further clarify the relationship between these two conditions.

\section{METHODS}

\section{The Third National Health and Nutrition Examination Survey}

The NHANES III (1988-1994) is a complex, multistage, stratified, clustered, probability sample study designed to obtain information regarding nutritional status and health of the US population. ${ }^{14}$ The survey included questionnaires, physical examination, and laboratory tests. The study was approved by the Center for Disease Control and Prevention's Institutional Review board. 


\section{Study population}

During the survey period, 16,115 subjects who were 20-74 years old completed the interview, physical examination, laboratory tests, and liver ultrasound examination. After excluding subjects without ultrasound images and those with ungradable degree of hepatic steatosis from ultrasound ( $\mathrm{n}=2259$ ), a total of 13,856 subjects were eligible for further study. We also excluded 2852 subjects with hepatic conditions other than NAFLD (ie, alcoholic liver disease, viral hepatitis, iron overload) and one subject without smoking status information. A total of 11,003 participants constituted the study cohort (figure 1).

\section{Data collection}

Baseline demographic data on age, race or ethnicity, sex, income, and education level were obtained using a questionnaire. Information on alcohol consumption, smoking, underlying medical conditions, physical activity, and drug use was obtained in a household interview. During the physical examination, height and body weight were measured and BMI was calculated. Blood pressure was recorded either at the household interview or during physical examination.

Race or ethnicity was categorized as 'non-Hispanic blacks', 'non-Hispanic whites', 'Mexican-American', and 'others'. Education was categorized as $\$ 8,9$ to 12, and $\geq 12$ years, based on completed years of schooling. Economic status was categorized as $\$ \$ 15,000$, $\$ 15,001$ to $\$ 25,000$, and $\$ 25,000$, according to the household income.

Smoking status was categorized as 'never', 'current', and 'former'. Current smokers were those who smoked daily or occasionally and reported having smoked more than 100 cigarettes in their lifetime. Former smokers were defined as those who reported having smoked >100 cigarettes during their lifetime but no longer smoked at all at the time of enrollment. Those who smoked $<100$ cigarettes during their lifetime were classified as 'having never smoked'. ${ }^{15}$ Heavy alcohol use was defined as an average of $>20 \mathrm{~g} /$ day for men and $>10 \mathrm{~g} /$ day for women. ${ }^{16}$

Physical activity was calculated based on a self-reported history of participating in one of the following activities during the past month: swimming, jogging, walking, running, aerobics, bicycling, weight lifting, and any gardening/yard work, dancing, and calisthenics. Based on the information, a physical activity intensity score was calculated using the ratio of metabolic rate achieved during physical activity compared with that during resting. The physical activity intensity score was then categorized as inactive (score $\$ 3.5$ ), moderately active (score 3.6-14.9), and active (score $\geq 15.0$ ). ${ }^{17}$

Insulin resistance was defined by a homeostasis of model assessment score >3.0. ${ }^{18}$ Elevated serum aminotransferases was defined as alanine aminotransferase (ALT) $>40 \mathrm{U} / \mathrm{L}$ or aspartate aminotransferase (AST) $>37 \mathrm{U} / \mathrm{L}$ (for men), and ALT or AST $>31 \mathrm{U} / \mathrm{L}$ (for women). Transferrin saturation was considered to be elevated when its level was $250 \%{ }^{19}$ Serum cotinine levels were measured using an isotope dilution, liquid chromatography, and tandem mass spectrometry method. The limit of detection using this method is $0.05 \mathrm{ng} / \mathrm{mL}$. Subjects with serum cotinine levels below the limit of detection were assigned a level of 
$0.035 \mathrm{ng} / \mathrm{mL}$. In our study, serum cotinine levels were determined and divided into quartiles for each smoking status (never-smokers, former smokers, and current smokers).

\section{Definitions of NAFLD and controls}

Ultrasonography examinations of the liver and gallbladder were performed in participants aged 20-74 years with a Toshiba (Tustin, California, USA) SSA-90A machine using a 3.75 and 5.0 MHz transducer. ${ }^{13}$ The liver was graded as normal, mild, moderate, or severe hepatic steatosis based on the following information: (1) Degree of the brightness of liver parenchyma, (2) Presence of liver-to-kidney contrast, (3) Presence of echogenic walls in the small intrahepatic vessels, (4) Presence of deep beam attenuation, and (5) Definition of the gallbladder walls. ${ }^{13}$

In the absence of a consensus definition, NAFLD in our study was defined as the presence of mild-to-severe hepatic steatosis on ultrasonography without other causes of chronic liver diseases (such as iron overload, defined by transferrin saturation $\geq 50 \%$, positive hepatitis B and $\mathrm{C}$ serology, and heavy alcohol consumption). Healthy controls were defined as those without any evidence of steatosis on ultrasonography and any evidence of chronic liver diseases.

\section{Statistical analyses}

All analyses were weighted to account for unequal probabilities of selection, based on the complexity of the survey data and the study design. Demographic and clinical differences among study participants according to smoking status were compared using the one-way analysis of variance or $\chi^{2}$ test, as appropriate. Serum cotinine was log-transformed to reduce the skewness in its distribution. Unconditional multivariate logistic regression was used to assess the association between smoking status/serum cotinine and NAFLD. The first model included age, sex, ethnicity, socioeconomic status, BMI, and physical activity. The second model included age, sex, ethnicity, socioeconomic status, BMI, physical activity, insulin resistance, systolic/diastolic blood pressure, and serum triglycerides and HDL. We also conducted stratified analyses based on self-reported smoking status. All analyses were performed using SAS V.9.3 (SAS Institute, Cary, North Carolina, USA). A p value $₫ \mathbf{\$} .05$ (two-sided) was considered statistically significant.

\section{RESULTS}

\section{Demographic and clinical characteristics of the study participants}

The schematic diagram on the selection of participants is shown in figure 1. Of the 11,003 participants included in the main analyses, $4036(37 \%)$ were identified as having NAFLD and $6967(63 \%)$ as controls. Subjects with NAFLD were older (48.5 vs 43 years, $\mathrm{p}<0.01)$, had higher systolic (129.1 vs $121.6 \mathrm{~mm} \mathrm{Hg}, \mathrm{p}<0.01)$ and diastolic $(77.1$ vs $73.5 \mathrm{~mm} \mathrm{Hg}$, $\mathrm{p}<0.01$ ) blood pressures; higher serum triglycerides ( $2.2 \mathrm{vs} 1.5 \mathrm{mmol} / \mathrm{L}, \mathrm{p}<0.01$ ), higher total cholesterol ( 5.5 vs $5.3 \mathrm{mmol} / \mathrm{L}, \mathrm{p}<0.01)$, higher circulating glycosylated hemoglobin (6.0\% vs 5.5\%, p<0.01), higher serum AST (24.4 vs $20.1 \mathrm{U} / \mathrm{L}, \mathrm{p}<0.01$ ), and higher serum ALT ( 23.5 vs $15.7 \mathrm{U} / \mathrm{L}, \mathrm{p}<0.01)$. Subjects with NAFLD were more likely to be men $(53.1 \%$ vs $41.6 \%, \mathrm{p}<0.01)$, obese $(49.5 \%$ vs $22.4 \%$, p $<0.01)$, and Mexican-American $(38.5 \%$ vs 
$28 \%, \mathrm{p}<0.01)$. Selected demographic and clinical characteristics stratified by smoking status are summarized in table 1 . Severe hepatic steatosis was found in 7\%, 9.9\%, and 5.8\% among non-smokers, former smokers, and current smokers, respectively $(\mathrm{p}<0.01)$.

\section{Association between smoking status, serum cotinine levels and NAFLD}

Using the questionnaires, $51.1 \%$ and $27.5 \%$ of NAFLD subjects were non-smokers and former smokers, respectively; whereas $53.1 \%$ of controls were non-smokers and $20.9 \%$ were former smokers. The percentage of current smokers was significantly lower in subjects with NAFLD compared with those in controls $(21.5 \%$ vs $26.0 \%, \mathrm{p}<0.01)$. The medians of serum cotinine levels were $0.32 \mathrm{ng} / \mathrm{mL}$ in those with NAFLD and $0.37 \mathrm{ng} / \mathrm{mL}$ in controls ( $\mathrm{p}<0.01$; table 2).

Associations of self-reported smoking status and serum cotinine levels with NAFLD are summarized in table 2. After adjustment for potential confounders in the model 1, there was no association between current (OR 0.92, 95\% CI 0.83 to 1.03) and former smokers (OR $1.11,95 \%$ CI 0.99 to 1.24 ) with NAFLD. No associations were observed between the levels of serum cotinine (OR 1.000, 95\% CI 0.999 to 1.000) or its log-transformed levels (OR $0.991,95 \%$ CI 0.977 to 1.006 ) with NAFLD. Similar results were observed in the model 2 (table 2).

The association of serum cotinine divided by quartile levels and NAFLD, stratified by smoking status is shown in table 3. No association between serum cotinine levels at each quartile level and NAFLD was observed regardless of smoking status in the logistic regression analyses for both models. The trend analysis demonstrated the inverse association between serum cotinine levels and NAFLD among current smokers ( $\mathrm{p}$ for trend $<0.01$ ).

\section{DISCUSSION}

In this population-based study, we found no association between NAFLD with self-reported smoking status and serum cotinine level. Active tobacco smoking is a known risk factor for several chronic diseases such as cancer, cardiovascular disease, and diabetes. ${ }^{20-22}$ Smoking also has an impact on liver diseases as it has shown to be a risk factor for advanced fibrosis in those with hepatitis $\mathrm{C}$ infection. ${ }^{23}$ The long-term morbidity and mortality from chronic hepatitis $\mathrm{C}$ infection are expected to decline due to highly effective, direct-acting antiviral agents. ${ }^{24}$ However, the health and economic burdens from NAFLD will continue due to the worsening epidemics of obesity and MetS. ${ }^{25}$ Therefore, identification of risk-modifying factors associated with NAFLD is of the utmost importance, as it may have an impact on the natural course of this disease.

MetS, through a combination of unfavorable health factors such as dyslipidemia, visceral obesity, and hypertension, is strongly associated with NAFLD. Smoking has also been associated with MetS. ${ }^{5726}$ Tobacco use is associated with increasing waist circumference, dyslipidemia, and hypertension which suggests a role for smoking on insulin resistance. ${ }^{52728}$ A recent population-based study of 59,467 individuals showed that smoking is associated with a higher risk of MetS in both genders and in all BMI classes. ${ }^{7}$ Moreover, the increased risk was stronger for heavy smokers but not as significant among light and former smokers. ${ }^{7}$

J Investig Med. Author manuscript; available in PMC 2018 January 01. 
Given the link between smoking and MetS, a known risk factor for NAFLD, several previous studies attempted to determine the relationship between smoking and NAFLD. ${ }^{89} \mathrm{~A}$ study from China observed a positive association between active smoking and NAFLD. ${ }^{9}$ Furthermore, active tobacco smoking and an elevated BMI had a synergistic effect on NAFLD prevalence. ${ }^{9}$ However, another study from Mexico found contradicting results. ${ }^{8}$ It is important to note these studies used the self-reported questionnaires to determine the smoking status of the participants. In our study, we found self-reported former smokers had a mean serum cotinine level of $24.6 \mathrm{ng} / \mathrm{mL}$. Therefore, there exists the concern of reporting bias that participants may tend to report less than the amount they actually smoke. It is important to note that we observed that among current smokers, the prevalence of NAFLD was lower in subjects with higher levels of cotinine (OR $0.62,95 \%$ CI 0.49 to 0.79 ) in the crude analysis. This finding is likely due to significantly lower BMI among NAFLD subjects with serum cotinine in the fourth quartile range $\left(25.2 \pm 5.0 \mathrm{~kg} / \mathrm{m}^{2}\right)$ than that in the first quartile $\left(27.8 \pm 6.1 \mathrm{~kg} / \mathrm{m}^{2}, \mathrm{p}<0.01\right)$. However, in the multivariate analyses adjusting for potential confounders, we found no association between serum cotinine levels at each quartile level and NAFLD.

NHANES III provided an opportunity for detailed analysis on the relationships between tobacco smoking and NAFLD. First, this is a population-based study representing the US population. It included comprehensive information on anthropometric measurements, laboratory results, and abdominal ultrasound and, thus, allowed us to determine the presence of MetS as well as NAFLD. Additionally, the use of self-reported questionnaires with NHANES III provided objective evidence of smoking exposure with levels of serum cotinine, a metabolite of nicotine which can be used as a marker of smoke exposure due to its longer half-life than nicotine. ${ }^{12}$ In this population-based study, we found no association between NAFLD with self-reported smoking status and serum cotinine level. It is known that the overall lifestyle of smokers is generally different from that of non-smokers. ${ }^{29}$ Despite the adjustments for physical activity in our analyses, we still did not find an association between smoking exposure and NAFLD.

In conclusion, in this large US population-based study, we did not find a relationship between NAFLD and self-reported smoking status or serum cotinine levels. Despite the strength of the sample size and the study cohort representing the US population, we acknowledge several limitations. NHANES III does not provide histological data which limited our ability to further categorize NAFLD by histological subtypes. Furthermore, ultrasound is not the most sensitive modality to characterize steatosis or diagnose NAFLD. However, it is the most practical screening modality for a large population-based study when obtaining liver biopsies is not feasible. Though we failed to demonstrate smoking as a possible risk-modifying factor associated with NAFLD, tobacco smoking is a well-known risk factor for several chronic diseases such as cancer, cardiovascular disease, and diabetes. Thus, interventions to promote successful smoking cessation should still be implemented as preventative measures. 


\section{Acknowledgments}

Funding: This study was supported by US Department of Veterans Affairs (1I01CX000361), National Institutes of Health (R01 DK107682; R21AA024935; U01AA021840), and US Department of Defense (W81XWH-12-1-0497).

\section{References}

1. Chalasani N, Younossi Z, Lavine JE, et al. The diagnosis and management of non-alcoholic fatty liver disease: practice Guideline by the American Association for the Study of Liver Diseases, American College of Gastroenterology, and the American Gastroenterological Association. Hepatology. 2012; 55:2005-23. [PubMed: 22488764]

2. Marchesini G, Bugianesi E, Forlani G, et al. Nonalcoholic fatty liver, steatohepatitis, and the metabolic syndrome. Hepatology. 2003; 37:917-23. [PubMed: 12668987]

3. Marchesini G, Brizi M, Bianchi G, et al. Nonalcoholic fatty liver disease: a feature of the metabolic syndrome. Diabetes. 2001; 50:1844-50. [PubMed: 11473047]

4. Expert Panel on Detection, Evaluation, and Treatment of High Blood Cholesterol in Adults. Executive summary of the third report of the National Cholesterol Education Program (NCEP) expert panel on detection, evaluation, and treatment of high blood cholesterol in adults (Adult Treatment Panel III). JAMA. 2001; 285:2486-97. [PubMed: 11368702]

5. Sun K, Liu J, Ning G. Active smoking and risk of metabolic syndrome: a meta-analysis of prospective studies. PLoS ONE. 2012; 7:e47791. [PubMed: 23082217]

6. Clair C, Chiolero A, Faeh D, et al. Dose-dependent positive association between cigarette smoking, abdominal obesity and body fat: cross-sectional data from a population-based survey. BMC Public Health. 2011; 11:23. [PubMed: 21223575]

7. Slagter SN, van Vliet-Ostaptchouk JV, Vonk JM, et al. Associations between smoking, components of metabolic syndrome and lipoprotein particle size. BMC Med. 2013; 11:195. [PubMed: 24228807]

8. Chavez-Tapia NC, Lizardi-Cervera J, Perez-Bautista O, et al. Smoking is not associated with nonalcoholic fatty liver disease. World J Gastroenterol. 2006; 12:5196-200. [PubMed: 16937532]

9. Liu Y, Dai M, Bi Y, et al. Active smoking, passive smoking, and risk of nonalcoholic fatty liver disease (NAFLD): a population-based study in China. J Epidemiol. 2013; 23:115-21. [PubMed: 23399520]

10. Bodmer CW, MacFarlane IA, Flavell HJ, et al. How accurate is the smoking history in newly diagnosed diabetic patients? Diabetes Res Clin Pract. 1990; 10:215-20. [PubMed: 2073868]

11. Masson EA, MacFarlane IA, Priestley CJ, et al. Failure to prevent nicotine addition in young people with diabetes. Arch Dis Child. 1992; 67:100-2. [PubMed: 1739319]

12. Belin RM, Astor BC, Powe NR, et al. Smoke exposure is associated with a lower prevalence of serum thyroid autoantibodies and thyrotropin concentration elevation and a higher prevalence of mild thyrotropin concentration suppression in the third National Health and Nutrition Examination Survey (NHANES III). J Clin Endocrinol Metab. 2004; 89:6077-86. [PubMed: 15579761]

13. NHANES Ultrasound procedure manual. http://www.cdc.gov/nchs/data/nhanes/nhanes3/ hepatic_steatosis_ultrasound_procedures_manual.pdf (accessed 7 Jul 2016)

14. Analytic and Reporting Guidelines: The National Health and Nutrition Examination Survey (NHANES). http://www.cdc.gov/nchs/data/nhanes/nhanes_03_04/ nhanes_analyfic_guidelines_dec_2005.pdf (accessed 7 Jul 2016)

15. Centers for Disease Control and Prevention (CDC). Cigarette smoking-attributable morbidityUnited States, 2000. MMWR Morb Mortal Wkly Rep. 2003; 52:842-4. [PubMed: 12966360]

16. Lazo M, Hernaez R, Eberhardt MS, et al. Prevalence of nonalcoholic fatty liver disease in the United States: the Third National Health and Nutrition Examination Survey, 1988-1994. Am J Epidemiol. 2013; 178:38-45. [PubMed: 23703888]

17. Park YW, Zhu S, Palaniappan L, et al. The metabolic syndrome: prevalence and associated risk factor findings in the US population from the Third National Health and Nutrition Examination Survey, 1988-1994. Arch Intern Med. 2003; 163:427-36. [PubMed: 12588201] 
18. Matthews DR, Hosker JP, Rudenski AS, et al. Homeostasis model assessment: insulin resistance and beta-cell function from fasting plasma glucose and insulin concentrations in man.

Diabetologia. 1985; 28:412-19. [PubMed: 3899825]

19. Stepanova M, Rafiq N, Younossi ZM. Components of metabolic syndrome are independent predictors of mortality in patients with chronic liver disease: a population-based study. Gut. 2010; 59:1410-15. [PubMed: 20660697]

20. Willi C, Bodenmann P, Ghali WA, et al. Active smoking and the risk of type 2 diabetes: a systematic review and meta-analysis. JAMA. 2007; 298:2654-64. [PubMed: 18073361]

21. Jatoi NA, Jerrard-Dunne P, Feely J, et al. Impact of smoking and smoking cessation on arterial stiffness and aortic wave reflection in hypertension. Hypertension. 2007; 49:981-5. [PubMed: 17372029]

22. Botteri E, Iodice S, Bagnardi V, et al. Smoking and colorectal cancer: a meta-analysis. JAMA. 2008; 300:2765-78. [PubMed: 19088354]

23. Pessione F, Ramond MJ, Njapoum C, et al. Cigarette smoking and hepatic lesions in patients with chronic hepatitis C. Hepatology. 2001; 34:121-5. [PubMed: 11431742]

24. Bennett H, McEwan P, Sugrue D, et al. Assessing the long-term impact of treating hepatitis C virus (HCV)-infected people who inject drugs in the UK and the relationship between treatment uptake and efficacy on future infections. PLoS ONE. 2015; 10:e0125846. [PubMed: 25938458]

25. Ruhl CE, Everhart JE. Fatty liver indices in the multiethnic United States National Health and Nutrition Examination Survey. Aliment Pharmacol Ther. 2015; 41:65-76. [PubMed: 25376360]

26. Nakanishi N, Takatorige T, Suzuki K. Cigarette smoking and the risk of the metabolic syndrome in middle-aged Japanese Male office workers. Ind Health. 2005; 43:295-301. [PubMed: 15895844]

27. Chiolero A, Faeh D, Paccaud F, et al. Consequences of smoking for body weight, body fat distribution, and insulin resistance. Am J Clin Nutr. 2008; 87:801-9. [PubMed: 18400700]

28. Chen CC, Li TC, Chang PC, et al. Association among cigarette smoking, metabolic syndrome, and its individual components: the metabolic syndrome study in Taiwan. Metab Clin Exp. 2008; 57:544-8. [PubMed: 18328358]

29. Rabaeus M, Salen P, de Lorgeril M. Is it smoking or related lifestyle variables that increase metabolic syndrome risk? BMC Med. 2013; 11:196. [PubMed: 24139143] 


\section{Significance of this study}

\section{What is already known about this subject?}

- Non-alcoholic fatty liver disease (NAFLD) is associated with obesity and metabolic syndrome.

- Several reports and a meta-analysis have shown that smoking is linked to metabolic syndrome.

- The association between smoking and NAFLD is debatable.

\section{What are the new findings?}

- In this large US population-based study, we did not find an association between NAFLD and self-reported smoking status or measured serum cotinine level.

\section{How might these results change the focus of research or clinical practice?}

- Though we failed to demonstrate smoking as a possible risk-modifying factor associated with NAFLD, tobacco smoking is a known risk factor for several chronic diseases such as cancer, cardiovascular disease, and diabetes. Thus, interventions should still be implemented to promote successful smoking cessation. 
NHANES III, age 20-74 years old Interview, examination, laboratory test, and ultrasound of the liver/gall bladder $(\mathrm{N}=16,115)$

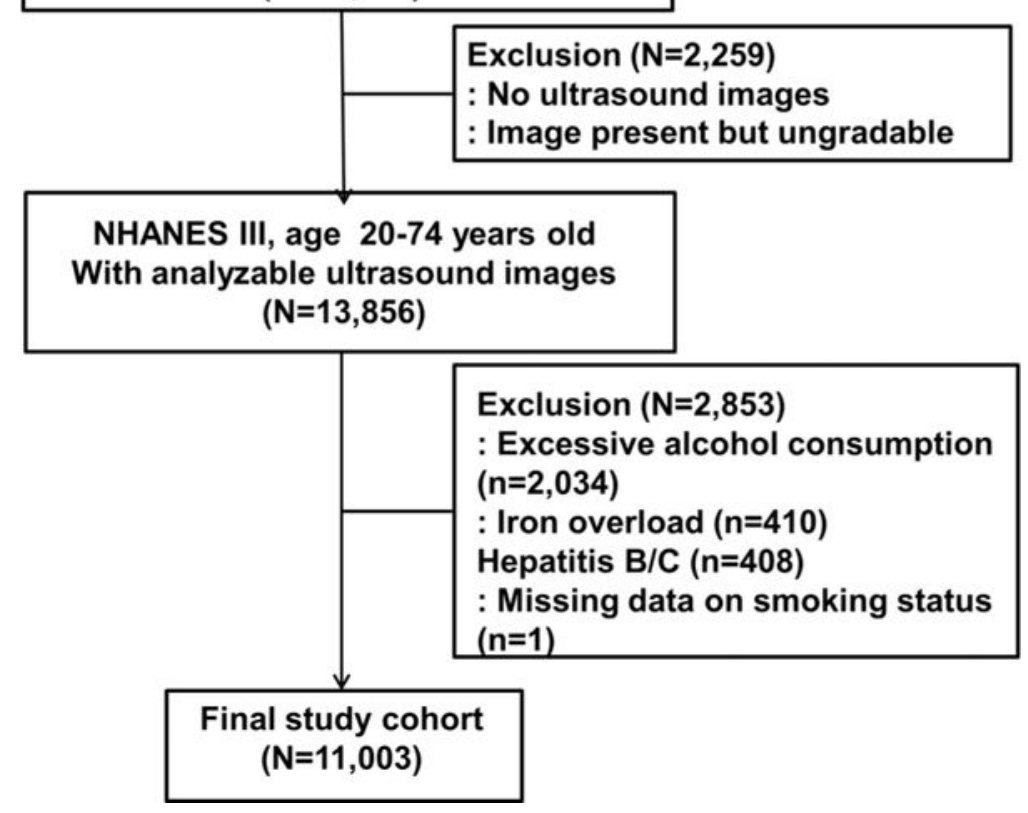

Figure 1.

Schematic diagram on the selection of the study participants. NHANES III, Third National Health and Nutrition Examination Survey. 
Table 1

Selected demographic and clinical characteristics of the study participants*

\begin{tabular}{|c|c|c|c|c|}
\hline & Non-smokers (N=5761) & Former smokers $(\mathrm{N}=\mathbf{2 5 6 3})$ & Current smokers ( $\mathrm{N}=\mathbf{2 6 7 9}$ ) & p Value ${ }^{\dagger}$ \\
\hline Age (years.) & $41.8(0.2)$ & $52.2(0.3)$ & $42.2(0.3)$ & $<0.01$ \\
\hline Men (n, \%) & $1833(31.8)$ & $1534(59.9)$ & $1344(50.2)$ & $<0.01$ \\
\hline \multicolumn{5}{|l|}{ Race or ethnicity (n, \%) } \\
\hline Non-Hispanic white & $1739(30.2)$ & $1227(47.9)$ & $986(36.8)$ & $<0.01$ \\
\hline Non-Hispanic black & $1726(30.0)$ & $555(21.7)$ & $940(35.1)$ & \\
\hline Mexican-American & $1979(34.4)$ & $706(27.5)$ & $664(24.8)$ & \\
\hline Other & $317(5.5)$ & $75(2.9)$ & $89(3.3)$ & \\
\hline \multicolumn{5}{|l|}{ Annual household income (n, \%) } \\
\hline$\$ \$ 15,000$ & $1670(32.1)$ & 649 (27.6) & $983(40.1)$ & $<0.01$ \\
\hline$\$ 15,001-\$ 25,000$ & $1205(23.1)$ & $551(23.5)$ & $598(24.4)$ & \\
\hline$>\$ 25,000$ & $2331(44.8)$ & $1148(48.9)$ & $871(35.5)$ & \\
\hline \multicolumn{5}{|l|}{ Education (n, \%) } \\
\hline$\$ 8$ years & $1289(22.5)$ & $636(24.9)$ & $614(23.1)$ & $<0.01$ \\
\hline $9-12$ years & $2604(45.5)$ & $1113(43.6)$ & $1527(57.5)$ & \\
\hline$>12$ years & $1828(32.0)$ & $801(31.4)$ & $516(19.4)$ & \\
\hline \multicolumn{5}{|l|}{ Body mass index (n, \%) } \\
\hline$<25 \mathrm{~kg} / \mathrm{m}^{2}$ & $2121(36.9)$ & $752(29.4)$ & $1181(44.1)$ & $<0.01$ \\
\hline $25-30 \mathrm{~kg} / \mathrm{m}^{2}$ & $1901(33.1)$ & $982(38.4)$ & $888(33.2)$ & \\
\hline $230 \mathrm{~kg} / \mathrm{m}^{2}$ & $1726(30.0)$ & $821(32.1)$ & $606(22.7)$ & \\
\hline \multicolumn{5}{|l|}{ Physical activity (n, \%) } \\
\hline Inactive & $1907(33.1)$ & $749(29.2)$ & $920(34.3)$ & $<0.01$ \\
\hline Moderately active & $2548(44.2)$ & $1300(50.7)$ & $1264(47.2)$ & \\
\hline Active & $1306(22.7)$ & $514(20.1)$ & $495(18.5)$ & \\
\hline Diabetes $F(\mathrm{n}, \%)$ & $777(13.5)$ & $456(17.8)$ & $356(13.3)$ & $<0.01$ \\
\hline Hypertension $\%$ (n, \%) & $1396(24.5)$ & $858(33.7)$ & $602(22.7)$ & $<0.01$ \\
\hline Hypercholesterolemia ${ }^{t}(\mathrm{n}, \%)$ & $928(32.5)$ & $656(40.7)$ & $383(34.9)$ & $<0.01$ \\
\hline Glycosylated hemoglobin (\%) & $5.5(0.02)$ & $5.8(0.03)$ & $5.6(0.02)$ & $<0.01$ \\
\hline Insulin resistance $\mathcal{S}_{(\mathrm{n}, \%)}$ & $2127(37.0)$ & $1102(43.0)$ & $847(31.7)$ & $<0.01$ \\
\hline Systolic BP (mm Hg) & $121.7(0.2)$ & $128.6(0.4)$ & $122.0(0.4)$ & $<0.01$ \\
\hline Diastolic BP (mm Hg) & $73.7(0.1)$ & $76.2(0.2)$ & $73.7(0.2)$ & $<0.01$ \\
\hline Serum triglycerides $(\mathrm{mmol} / \mathrm{L})$ & $1.5(0.02)$ & $1.9(0.03)$ & $1.7(0.03)$ & $<0.01$ \\
\hline Serum total cholesterol $(\mathrm{mmol} / \mathrm{L})$ & $5.2(0.02)$ & $5.6(0.02)$ & $5.2(0.02)$ & $<0.01$ \\
\hline Serum LDL cholesterol $(\mathrm{mmol} / \mathrm{L})$ & $3.3(0.02)$ & $3.5(0.03)$ & $3.3(0.03)$ & $<0.01$ \\
\hline Serum HDL cholesterol (mmol/L) & $1.3(0.005)$ & $1.3(0.008)$ & $1.2(0.008)$ & $<0.01$ \\
\hline Serum alanine aminotransferase (U/L) & $17.7(0.2)$ & $18.3(0.3)$ & $16.6(0.3)$ & $<0.01$ \\
\hline Serum aspartate aminotransferase (U/L) & $21.2(0.2)$ & $22.0(0.2)$ & $20.1(0.2)$ & $<0.01$ \\
\hline Serum $\gamma$-glutamyltransferase (U/L) & $28.3(0.5)$ & $33.6(0.9)$ & $32.2(0.9)$ & $<0.01$ \\
\hline Serum uric acid $(\mu \mathrm{mol} / \mathrm{L})$ & $300.4(1.2)$ & $335.0(1.8)$ & $310.1(1.7)$ & $<0.01$ \\
\hline
\end{tabular}




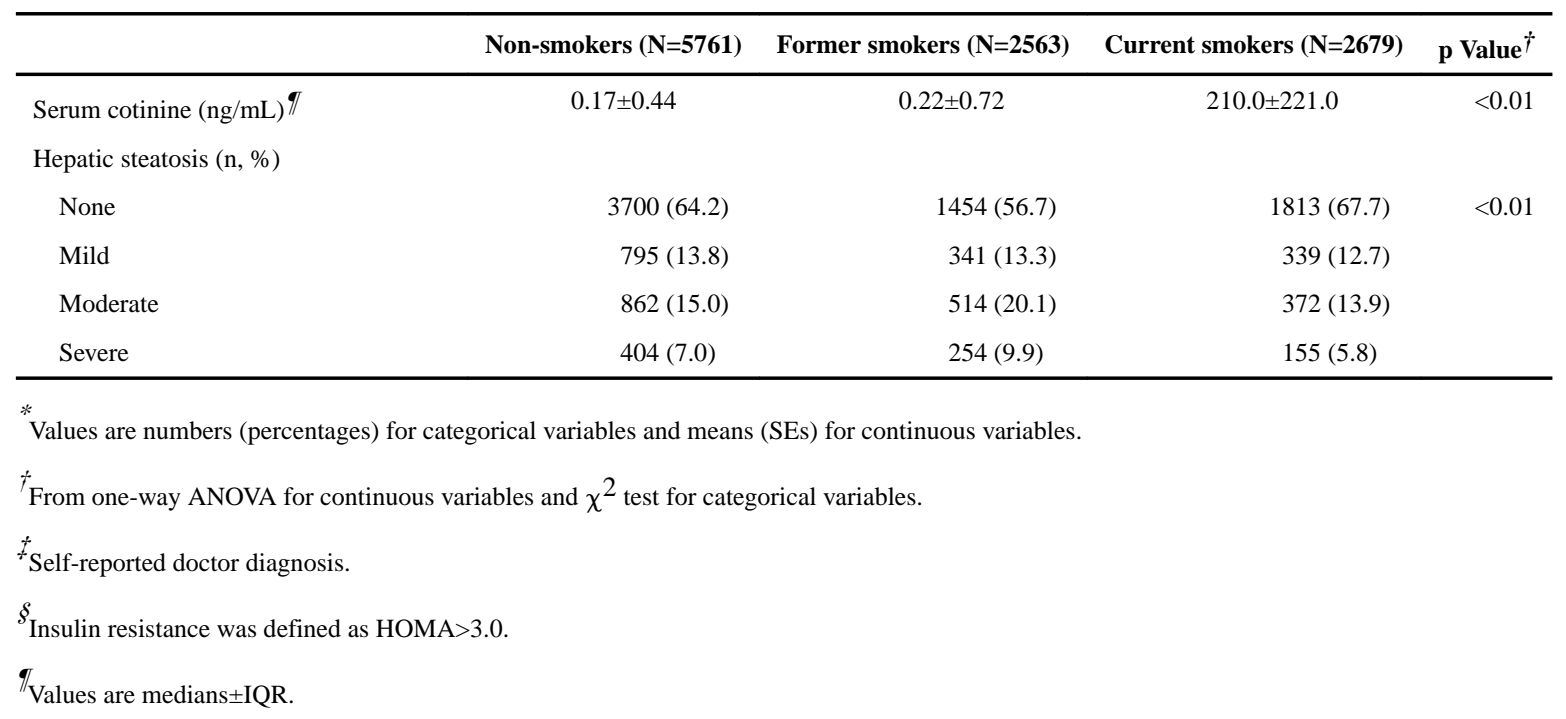

ANOVA, analysis of variance; BP, blood pressure; HDL, high-density lipoprotein; HOMA, homeostasis of model assessment score; LDL, lowdensity lipoprotein. 


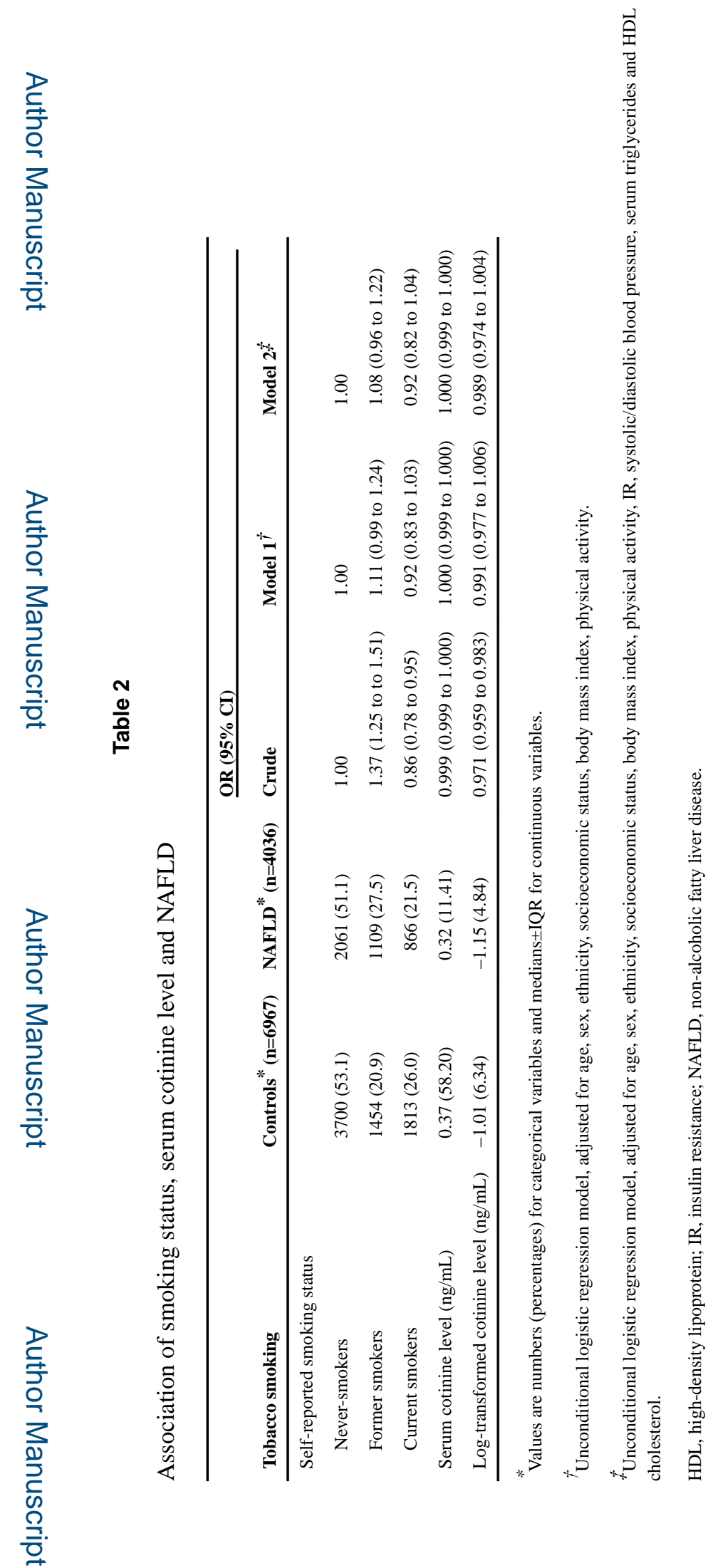

J Investig Med. Author manuscript; available in PMC 2018 January 01. 


\section{롤 \\ ํㅗㄹ}

\section{新}

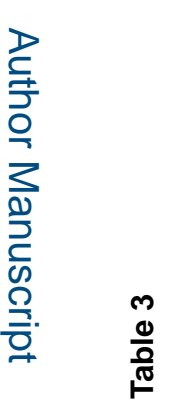

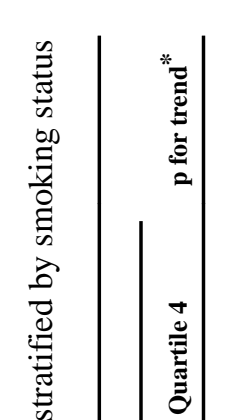

용

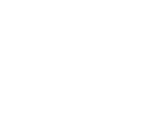

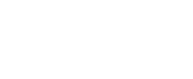

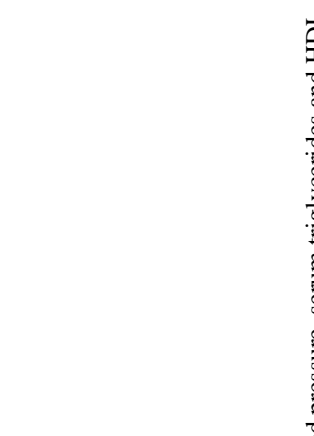

崖

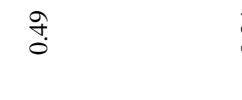

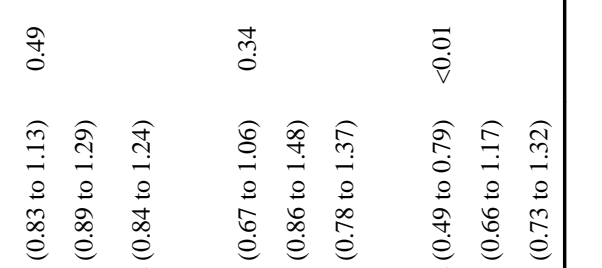

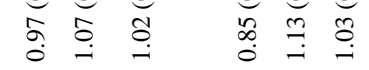

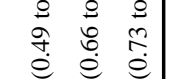

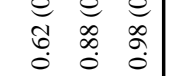

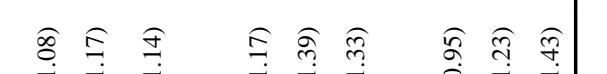

定

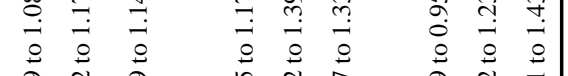

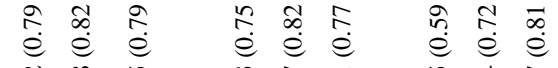

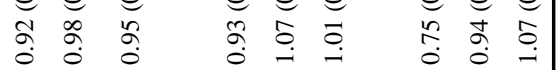

를

疍

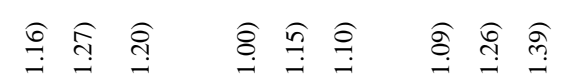

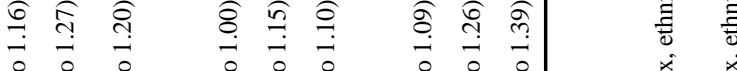

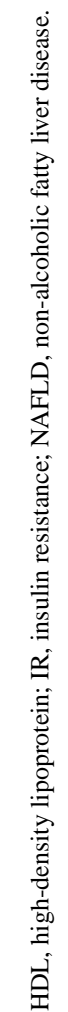

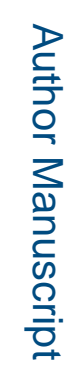

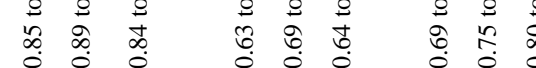

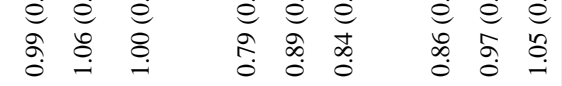

요 -

s

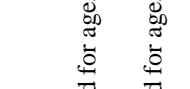

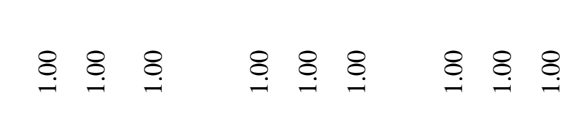

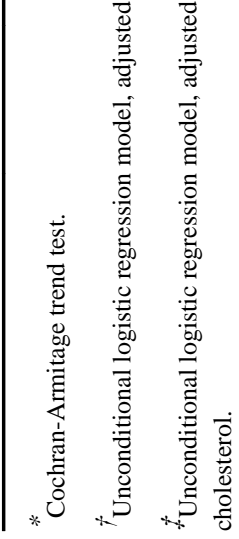

